\title{
Calidad de la canal y la carne en pollos de ceba que consumen Roystonea regia
}

Madeleidy Martínez-Pérez ${ }^{1 *} \bowtie(\mathbb{0})$ Ph.D; Yesenia Vives-Hernández ${ }^{1}$ 凶(0) Lic; Bárbara Rodríguez $\mathrm{S}^{1}{ }^{凶(1)}$ Ph.D; Osney Pérez-Acosta ${ }^{\circledR(0)}$ M.Sc.

${ }^{1}$ Instituto de Ciencia Animal, Km 47 1/2 Carretera Central, San José de Las Lajas, Mayabeque, Cuba.

*Correspondencia: mademar@ica.co.cu

Recibido: Mayo 2020; Aceptado: Noviembre 2020; Publicado: Marzo 2021.

\section{RESUMEN}

Objetivo. Estudiar la calidad de la canal y la carne en pollos de ceba que consumen harina de frutos de Roystonea regia (palmiche). Materiales y métodos. Se utilizaron 40 pollos de ceba distribuidos según diseño completamente aleatorizado en cuatro tratamientos: control (maíz-pasta de soya) y la inclusión de 5, 10 y $15 \%$ de harina de palmiche. Se estudió el rendimiento de la canal, grasa abdominal y la calidad de la carne (tecnológicos y composición de ácidos grasos). Para los parámetros de apariencia se realizó análisis de varianza no paramétrico de clasificación simple. Resultados. No se encontraron diferencias entre tratamientos para el peso de la canal y pH a los 45 minutos y 24 horas postmortem. Hubo reducción de la grasa abdominal con 5 y $15 \%$ respecto al control (13.00 y 11.96 vs $17.02 \mathrm{~g} / \mathrm{kg})$, en tanto que el $10 \%$ no difirió del resto $(15.10 \mathrm{~g} / \mathrm{kg})(\mathrm{p}<0.01)$. No se encontraron diferencias en el color, excepto para la luminosidad en el muslo que fue superior con la inclusión de 10 y $15 \%$ (48.62 y 49.22$)$ respecto al $5 \%(45.32)$ y el control no difirió entre tratamientos (47.53) $(p<0.05)$. La composición de ácidos grasos en las diferentes porciones comestibles mostró que las aves depositaron los ácidos oleico, linoleico y palmítico en mayor concentración. Conclusiones. La inclusión hasta el $15 \%$ de harina de palmiche a las dietas de pollos de ceba no modifica el rendimiento de la canal, reduce la grasa abdominal e incide positivamente en los indicadores de calidad de la carne.

Palabras clave: Aves; ácidos grasos; carnes; palma (Fuente: CAB thesaurus).

\section{ABSTRACT}

Objective. To study meat and carcass quality in broilers fed Roystonea regia (royal palm nut) fruits meals. Materials and methods. A total of 40 broilers distributed according to a completely randomized design were used in four treatments: control (corn-soybean paste) and the inclusion of 5, 10 and $15 \%$ royal palm nut meal. The carcass yield, abdominal fat and meat quality (technological and fatty acid composition (FA)) were studied. For the appearance parameters, a non-parametric analysis of simple classification was performed. Results. There were no differences between treatments for carcass weight and $\mathrm{pH}$ at 45 minutes and 24 hours postmortem. There was a reduction in abdominal fat by 5 and $15 \%$ compared to the control (13.00 and 11.96 vs. $17.02 \mathrm{~g} / \mathrm{kg}$ ), while $10 \%$ did not differ from the rest $(15.10 \mathrm{~g} / \mathrm{kg})(p<0.01)$. There were no differences in color, except for the luminosity in the drumstick that was superior with the inclusion of 10 and $15 \%$ (48.62 and 49.22) with respect

Como citar (Vancouver).

Martínez-Pérez M, Vives-Hernández Y, Rodríguez SB, Pérez-Acosta O. Calidad de la canal y la carne en pollos de ceba que consumen Roystonea regia. Rev MVZ Córdoba. 2021; 26(2):e1984. https://doi.org/10.21897/rmvz.1984 
to $5 \%(45.32)$ and the control did not differ between treatments $(47.53)(p<0.05)$. The composition of FA in the different edible portions showed that the broilers deposited oleic, linoleic and palmitic acids in higher proportions. Conclusions. The inclusion of royal palm nut meal in broiler diets does not change the carcass yield, reduces abdominal fat and positively affects meat quality indicators.

Keywords: Poultry; fatty acids; meats; palm (Source: CAB thesaurus).

\section{INTRODUCCIÓN}

La carne de aves se ajusta a la demanda actual de los consumidores por ser baja en grasa, con alto grado de insaturación en sus ácidos grasos (AG) y niveles bajos de sodio y colesterol. También se puede considerar como un "alimento funcional", que proporciona sustancias bioactivas con efectos favorables en la salud humana, como el ácido linoleico conjugado, vitaminas y antioxidantes y una razón equilibrada de n-6 a n-3 (1). Sin embargo, para que se logren estas características, es primordial el uso de fuentes alimenticias con buen balance de AG.

El subtrópico cubano es el hábitat natural de palmas y encinos, que son fuentes de grasa localmente disponibles, los que se utilizan tradicionalmente en la cría del ganado porcino (2). Sin embargo, aunque diferentes nutricionistas suponen acerca de su inclusión en la dieta de las aves, es poco lo que se conoce. Según Rodríguez et al (3) y Marrero et al (4) el aceite del fruto entero de la palma real (Roystonea regia) conocido como palmiche, es más rico en AG insaturados, por lo que pudiera ser una buena fuente de lípidos para la carne aviar.

De todos los atributos de calidad, la apariencia es la más crítica para la selección de muchos alimentos. Los consumidores a menudo seleccionan o rechazan un producto basados únicamente en su apariencia ya que ésta a menudo afecta a otras propiedades sensoriales. Desde hace tiempo se sabe que el color es un importante criterio de selección para aves frescas y productos cárnicos, así como para la satisfacción del producto final (5). Es por ello, que el objetivo de este trabajo fue estudiar indicadores de calidad de la canal y la carne de pollos de ceba que consumen harina de frutos de Roystonea regia (palmiche).

\section{MATERIALES Y MÉTODOS}

Animales y dietas. Se utilizaron 40 pollos de ceba machos (híbrido $\mathrm{HE}_{21}$ ) de peso promedio $704.2 \pm 27.78 \mathrm{~g}$ y edad 8 días. Se alojaron de manera aleatoria en jaulas individuales para metabolismo de alambre galvanizado cuyas dimensiones fueron de $40 \times 40 \times 40 \mathrm{~cm}$. Durante todo el tiempo de experimentación los animales tuvieron libre acceso al agua y al alimento.

Los tratamientos experimentales consistieron en cuatro dietas experimentales para inicio, crecimiento y acabado según los requerimientos de la NRC (6): Control (maíz-pasta de soya) y la inclusión de 5, 10 y $15 \%$ de harina de palmiche. Estas se presentan en las tablas 1, 2 y 3, respectivamente. Las dietas se elaboraron semanalmente para evitar la pérdida de calidad de la harina de palmiche.

Obtención de la harina de palmiche. El grano de palmiche se obtuvo de diferentes palmares de la provincia Mayabeque, Cuba. Después de cortadas las ramas del mismo, se colocaron al sol hasta su maduración. Posteriormente, se extendieron en plato de secado hasta alcanzar un color café-parduzco y se almacenaron en sacos hasta el momento de su utilización. De esta manera se evitó el enranciamiento que el contenido de aceite pudiera ocasionar. Este proceso se realizó en las instalaciones de la Unidad Integral Avícola del Instituto de Ciencia Animal. En el momento de su utilización para la elaboración de las dietas experimentales, los granos secos, se molieron en molino de martillo (JF 2D, Brasil) a tamaño de partículas de $3 \mathrm{~mm}$ y posteriormente se mezclaron con el resto de los ingredientes del alimento.

Procedimiento experimental. El experimento se realizó en las instalaciones del Instituto de Ciencia Animal, Mayabeque, Cuba. 
Tabla 1. Dietas experimentales correspondientes al periodo de inicio (8-21 días).

\begin{tabular}{|c|c|c|c|c|}
\hline Ingrediente & Control & Palmiche 5\% & Palmiche $10 \%$ & Palmiche 15\% \\
\hline Harina Maíz & 48.60 & 43.00 & 39.00 & 32.50 \\
\hline Harina Soya & 41.08 & 41.37 & 40.74 & 41.74 \\
\hline Aceite Vegetal & 6.00 & 6.40 & 6.40 & 6.75 \\
\hline Fosfato Mono cálcico & 1.80 & 1.65 & 1.52 & 1.40 \\
\hline Carbonato Calcio & 1.50 & 1.55 & 1.56 & 1.55 \\
\hline Sal Común & 0.35 & 0.35 & 0.35 & 0.35 \\
\hline DL-metionina & 0.24 & 0.13 & 0.13 & 0.13 \\
\hline Colina & 0.13 & 0.25 & - & 0.28 \\
\hline Premezcla minero vitamínica ${ }^{1}$ & 0.30 & 0.30 & 0.30 & 0.30 \\
\hline Harina de Palmiche & 0 & 5 & 10 & 15 \\
\hline \multicolumn{5}{|c|}{ Composición calculada \% } \\
\hline $\mathrm{PB}$ & 21.96 & 21.94 & 22.00 & 21.96 \\
\hline $\mathrm{EM}, \mathrm{kJ} / \mathrm{kg}$ & 12.95 & 12.94 & 12.92 & 12.83 \\
\hline FB & 3.04 & 4.47 & 5.87 & 7.32 \\
\hline $\mathrm{Pd}$ & 0.50 & 0.50 & 0.50 & 0.50 \\
\hline $\mathrm{Ca}$ & 1.00 & 1.00 & 1.00 & 1.00 \\
\hline Met. +Cys. & 0.890 & 0.880 & 0.880 & 0.880 \\
\hline Lisina & 1.22 & 1.22 & 1.22 & 1.22 \\
\hline
\end{tabular}

${ }^{1}$ Suplemento vitamínico: vitam. A, 10 000UI; vitam. D3, 2000 UI; vitam. E, 10 mg; vitam. K, 2 mg; tiamina, 1 mg; riboflavina, $5 \mathrm{mg}$; piridoxina, $2 \mathrm{mg}$; vitam. B12, $15.4 \mu \mathrm{g}$; ácido nicotínico, $125 \mathrm{mg}$; pántotenato de calcio, $10 \mathrm{mg} ;$ ácido fólico, $0.25 \mathrm{mg}$; biotina, $0.02 \mathrm{mg}$. Suplemento mineral: selenio, $0.1 \mathrm{mg}$; hierro, $40 \mathrm{mg}$; cobre, $12 \mathrm{mg}$; zinc, $120 \mathrm{mg}$; magnesio, $100 \mathrm{mg}$; iodo, $2.5 \mathrm{mg}$; cobalto $0.75 \mathrm{mg}$.

Tabla 2. Dietas experimentales correspondientes al periodo de crecimiento (22-35 días).

\begin{tabular}{|c|c|c|c|c|}
\hline Ingrediente & Control & Palmiche 5\% & Palmiche 10\% & Palmiche 15\% \\
\hline Harina Maíz & 55.40 & 50.29 & 44.65 & 38.92 \\
\hline Harina Soya & 35.21 & 35.00 & 35.25 & 35.55 \\
\hline Aceite Vegetal & 5.50 & 5.80 & 6.15 & 6.55 \\
\hline Fosfato Mono cálcico & 1.58 & 1.60 & 1.60 & 1.63 \\
\hline Carbonato Calcio & 1.40 & 1.40 & 1.40 & 1.38 \\
\hline Sal Común & 0.30 & 0.30 & 0.30 & 0.30 \\
\hline Metionina & 0.18 & 0.18 & 0.13 & 0.13 \\
\hline Cloruro de colina & 0.13 & 0.13 & 0.22 & 0.24 \\
\hline Premezcla minero vitamínica 1 & 0.30 & 0.30 & 0.30 & 0.30 \\
\hline Harina de palmiche & 0 & 5 & 10 & 15 \\
\hline \multicolumn{5}{|c|}{ Composición calculada, \% } \\
\hline PB & 19.87 & 19.72 & 19.72 & 19.74 \\
\hline $\mathrm{EM}, \mathrm{kJ} / \mathrm{kg}$ & 13.17 & 13.15 & 13.12 & 13.10 \\
\hline FB & 2.84 & 4.25 & 5.67 & 7.10 \\
\hline $\mathrm{Pd}$ & 0.45 & 0.45 & 0.45 & 0.45 \\
\hline $\mathrm{Ca}$ & 0.91 & 0.92 & 0.92 & 0.93 \\
\hline Met. +Cys. & 0.780 & 0.776 & 0.782 & 0.781 \\
\hline Lisina & 1.07 & 1.06 & 1.05 & 1.05 \\
\hline
\end{tabular}

'Suplemento vitamínico: vitam. A, 10 000UI; vitam. D3, 2000 UI; vitam. E, 10 mg; vitam. K, 2 mg; tiamina, 1 mg; riboflavina, $5 \mathrm{mg}$; piridoxina, $2 \mathrm{mg}$; vitam. B12, $15.4 \mu \mathrm{g}$; ácido nicotínico, $125 \mathrm{mg}$; pantotenato de calcio, $10 \mathrm{mg} ;$ ácido fólico, $0.25 \mathrm{mg}$; biotina, $0.02 \mathrm{mg}$. Suplemento mineral: selenio, $0.1 \mathrm{mg}$; hierro, $40 \mathrm{mg}$; cobre, $12 \mathrm{mg}$; zinc, 120 mg; magnesio, $100 \mathrm{mg}$; iodo, $2.5 \mathrm{mg}$; cobalto $0.75 \mathrm{mg}$.

Tabla 3. Dietas experimentales correspondientes al periodo de acabado (36-42 días).

\begin{tabular}{|c|c|c|c|c|}
\hline Ingrediente & Control & Palmiche 5\% & Palmiche 10\% & Palmiche $15 \%$ \\
\hline Harina Maíz & 60.52 & 55.51 & 50.36 & 45.49 \\
\hline Harina Soya & 30.12 & 30.18 & 30.28 & 30.15 \\
\hline Aceite Veqetal & 5.40 & 5.50 & 5.70 & 5.85 \\
\hline Fosfato Mono cálcico & 1.50 & 1.35 & 1.25 & 1.20 \\
\hline Carbonato Calcio & 1.50 & 1.50 & 1.45 & 1.35 \\
\hline Sal Común & 0.35 & 0.35 & 0.35 & 0.35 \\
\hline Metionina & 0.18 & 0.18 & 0.18 & 0.18 \\
\hline Cloruro de colina & 0.13 & 0.13 & 0.13 & 0.13 \\
\hline Premezcla minero vitamínica ${ }^{1}$ & 0.30 & 0.30 & 0.30 & 0.30 \\
\hline Harina de palmiche & 0 & 5 & 10 & 15 \\
\hline \multicolumn{5}{|c|}{ Composición calculada, \% } \\
\hline $\mathrm{PB}$ & 18.03 & 18.00 & 17.98 & 17.88 \\
\hline $\mathrm{EM}, \mathrm{kJ} / \mathrm{kg}$ & 13.39 & 13.33 & 13.30 & 13.25 \\
\hline & 2.66 & 4.08 & 5.50 & 6.92 \\
\hline $\mathrm{Pd}$ & 0.43 & 0.43 & 0.43 & $0.4 \overline{3}$ \\
\hline $\mathrm{Ca}$ & 0.92 & 0.93 & 0.92 & 0.91 \\
\hline Met.+Cys. & 0.732 & 0.727 & 0.718 & 0.718 \\
\hline Lisina & 0.941 & 0.935 & 0.936 & 0.936 \\
\hline
\end{tabular}

1Suplemento vitamínico: vitam. A, 10 000UI; vitam. D3, 2000 UI; vitam. E, 10 mg; vitam. K, 2 mg; tiamina, 1 mg; riboflavina $5 \mathrm{mg}$; piridoxina, $2 \mathrm{mg}$; vitam. B12, $15.4 \mu \mathrm{g}$; ácido nicotínico, $125 \mathrm{mg}$; pantotenato de calcio, $10 \mathrm{mg}$; ácido fólico, $0.25 \mathrm{mg}$; biotina, $0.02 \mathrm{mg}$. Suplemento mineral: selenio, $0.1 \mathrm{mg}$; hierro, $40 \mathrm{mg}$; cobre, $12 \mathrm{mg}$; zinc, $120 \mathrm{mg}$; magnesio, $100 \mathrm{mg}$; iodo, $2.5 \mathrm{mg}$; cobalto $0.75 \mathrm{mg}$. 
A los 42 días, los animales se pesaron y se sacrificaron dos horas y treinta minutos después de la ingestión de alimento. Fueron aturdidos con descarga eléctrica y seguidamente desangrados por la vena yugular. Posteriormente, se abrió la cavidad abdominal, se evisceraron y se separaron manualmente la piel y las plumas. Se pesaron las canales y la grasa abdominal en una balanza técnica (SARTORIUS, Alemania). Para el análisis estadístico, los pesos se expresaron como relativos al peso vivo ( $\mathrm{PV}, \mathrm{g} / \mathrm{kg})$.

Indicadores de calidad de la carne. Se determinaron los indicadores tecnológicos y de apariencia en las principales porciones comestibles: muslo, pierna, pechuga y grasa abdominal.

Tecnológicos. Se midió $\mathrm{pH}$ con $\mathrm{pH}$ metro digital (HANNA, Rumanía) a los 45 minutos posterior al sacrificio. Seguidamente, se conservaron en refrigeración a $4^{\circ} \mathrm{C}$ hasta las 24 horas, donde se midió el indicador nuevamente. Posteriormente, se separó la carne del hueso en cada una de las porciones para determinar los parámetros de apariencia y composición de ácidos grasos.

Apariencia. Se determinó el color a partir de las variables cualitativas L* (luminosidad), a* (pigmentos rojos) y $b^{*}$ (pigmentos amarillos). Los análisis se realizaron por triplicado en cada una de las porciones, con colorímetro (Cromamater ${ }^{\circledR}$, USA) y escala CIELAB.

Composición de ácidos grasos. Se determinó la concentración en la carne de láurico (12:0), mirístico (14:0), palmítico (16:0), palmitoleico $(16: 1)$, oleico $(18: 1)$, linoleico $(18: 2)$ y linolénico (18:3). Se realizaron mezclas de las diferentes porciones y la grasa abdominal de tres animales para conformar tres muestras por tratamiento. Los AG se determinaron como ésteres metílicos según el procedimiento descrito por Rodríguez et al (3). Se empleó un cromatógrafo de gases GC-14B (SHIMADZU, Japón), acoplado a sistema de cómputo, con detector de ionización por llama y una columna capilar BPX-70 (30 m x 0,53 $\mathrm{mm}, 1 \mu \mathrm{m}$ Df, SGE, Australia). El programa de temperatura fue de $1 \mathrm{~min}$ a $100^{\circ} \mathrm{C}$, de $100^{\circ} \mathrm{C}$ hasta $220^{\circ} \mathrm{C}$ a $10^{\circ} \mathrm{C} / \mathrm{min}$ y $2 \mathrm{~min}$ a $220^{\circ} \mathrm{C}$. La temperatura del detector y el inyector fue $220^{\circ} \mathrm{C}$, y el flujo del gas portador $\left(\mathrm{H}_{2}\right)$ fue $8,6 \mathrm{~mL} / \mathrm{min}$. Los análisis se realizaron por triplicado. Los patrones de AG (Sigma, USA) y los reactivos y disolventes (Merck, Alemania) fueron puros para análisis.
El análisis cuantitativo de los ácidos grasos se realizó por el método del patrón interno. Se identificaron inicialmente los ésteres metílicos en la muestra de ensayo, por comparación de la retención relativa de cada componente. Se calcularon las concentraciones respecto al tiempo de retención del patrón interno, con la retención relativa de cada éster metílico de referencia.

Métodos estadísticos. Se utilizó diseño completamente aleatorizado con cuatro tratamientos que consistieron en las dietas experimentales y diez repeticiones de un animal. Para el análisis de los indicadores rendimiento de la canal, grasa abdominal y los de calidad de la carne (tecnológicos y composición de ácidos grasos) los valores medios se compararon mediante la dócima de Duncan (7) para $p<0.05$, en los casos necesarios. Para los parámetros de apariencia se realizó análisis de varianza no paramétrico de clasificación simple y se aplicó dócima de Conover (8) para $p<0.05$, cuando fue preciso. Se empleó el programa estadístico computarizado INFOSTAT (9).

\section{RESULTADOS}

En la tabla 4 se muestran los resultados de los pesos vivo final y relativo de la canal, que no mostraron diferencias entre tratamientos. El peso de la grasa abdominal se redujo en los tratamientos 5 y $15 \%$ con respecto al control, en tanto que el $10 \%$ de palmiche no difirió del resto (Tabla 4).

Tabla 4. Pesos vivo final y relativo de la canal y la grasa abdominal de pollos de ceba que consumen harina de palmiche.

\begin{tabular}{|c|c|c|c|c|c|c|}
\hline \multirow{2}{*}{ Indicador } & \multicolumn{4}{|c|}{ Tratamientos } & \multirow{2}{*}{ EE \pm} & \multirow{2}{*}{ p-valor } \\
\hline & Control & P 5\% & P $10 \%$ & P $15 \%$ & & \\
\hline $\begin{array}{l}\text { Peso vivo } \\
\text { final }(\mathrm{kg})\end{array}$ & 2.24 & 2.36 & 2.34 & 2.15 & 0.15 & 0.7506 \\
\hline $\begin{array}{c}\text { Peso canal } \\
(\mathrm{g} / \mathrm{kg})\end{array}$ & 532.78 & 568.99 & 547.76 & 579.44 & 0.09 & 0.9646 \\
\hline $\begin{array}{c}\text { Grasa } \\
\text { abdominal } \\
(\mathrm{g} / \mathrm{kg})\end{array}$ & $17.02^{\mathrm{b}}$ & $13.00^{\mathrm{a}}$ & $15.10^{\mathrm{ab}}$ & $11.96^{a}$ & 1.08 & 0.0106 \\
\hline
\end{tabular}

No se observaron diferencias entre tratamientos para el pH en las diferentes secciones comestibles de la canal de pollos de ceba al incluir harina de palmiche en la ración a los 45 minutos y 24 horas postmortem (Tabla 5). 
Tabla 5. Comportamiento del $\mathrm{pH}$ en las diferentes secciones comestibles de pollos de ceba que consumen harina de palmiche postmortem.

\begin{tabular}{cccccccc}
\hline pH & \multicolumn{5}{c}{ Tratamientos } & EE & p-valor \\
\cline { 2 - 6 } & Control P 5\% P 10\% P 15\% & 10 & & \\
\hline $\begin{array}{c}\text { Muslo 45 } \\
\text { minutos }\end{array}$ & 6.35 & 6.44 & 6.37 & 6.34 & 1.09 & 0.3670 \\
$\begin{array}{c}\text { Muslo 24 } \\
\text { horas }\end{array}$ & 6.31 & 6.23 & 6.25 & 6.32 & 1.50 & 0.2317 \\
$\begin{array}{c}\text { Pierna 45 } \\
\text { minutos }\end{array}$ & 6.36 & 6.40 & 6.35 & 6.35 & 0.19 & 0.9054 \\
$\begin{array}{c}\text { Pierna 24 } \\
\text { horas }\end{array}$ & 6.23 & 6.33 & 5.95 & 6.35 & 1.08 & 0.3708 \\
$\begin{array}{c}\text { Pechuga 45 } \\
\text { minutos }\end{array}$ & 6.23 & 6.17 & 6.20 & 5.83 & 1.24 & 0.3087 \\
$\begin{array}{c}\text { Pechuga 24 } \\
\text { horas }\end{array}$ & 6.05 & 6.03 & 5.98 & 6.01 & 0.48 & 0.6971 \\
\hline
\end{tabular}

$\mathrm{P}=$ Palmiche.

En la tabla 6 se muestran los resultados de los diferentes pigmentos en las secciones comestibles de pollos de ceba. No se observaron diferencias para los indicadores, excepto para la luminosidad en el muslo que fue superior con la inclusión de 10 y $15 \%$ de harina de palmiche en la ración $(p<0.05)$ con respecto al $5 \%$ y el control no difirió entre tratamientos.

Tabla 6. Parámetros de coloración en las diferentes secciones comestibles de pollos de ceba que consumen harina de palmiche después de 24 horas de sacrificio.

\begin{tabular}{|c|c|c|c|c|c|}
\hline \multirow{2}{*}{ Variables } & \multicolumn{4}{|c|}{ Tratamientos } & \multirow{2}{*}{ p-valor } \\
\hline & Control & P 5\% & P $10 \%$ & P 15\% & \\
\hline \multicolumn{6}{|c|}{ Muslo } \\
\hline$L^{*}$ & $\begin{array}{r}20.60^{\mathrm{ab}} \\
(47.53) \\
\mathrm{DE}=3.69\end{array}$ & $\begin{array}{c}12.05 \mathrm{a} \\
(45.32) \\
D E=2.23\end{array}$ & $\begin{array}{r}23.70^{\mathrm{b}} \\
(48.62) \\
\mathrm{DE}=2.90\end{array}$ & $\begin{array}{c}25.65^{b} \\
(49.22) \\
D E=3.26\end{array}$ & 0.0478 \\
\hline a* & $\begin{array}{c}24.90 \\
(9.50) \\
D E=9.57\end{array}$ & $\begin{array}{c}21.70 \\
(9.27) \\
D E=8.65\end{array}$ & $\begin{array}{c}19.10 \\
(8.92) \\
\mathrm{DE}=8.64\end{array}$ & $\begin{array}{c}16.30 \\
(8.26) \\
D E=8.16\end{array}$ & 0.3984 \\
\hline b* & $\begin{array}{c}16.90 \\
(2.57) \\
D E=2.35\end{array}$ & $\begin{array}{c}24.90 \\
(3.93) \\
\mathrm{DE}=4.03\end{array}$ & $\begin{array}{c}17.60 \\
(2.80) \\
\mathrm{DE}=3.34\end{array}$ & $\begin{array}{c}22.60 \\
(3.28) \\
\mathrm{DE}=4.05\end{array}$ & 0.3472 \\
\hline \multicolumn{6}{|c|}{ Pierna } \\
\hline$L^{*}$ & $\begin{array}{c}17.60 \\
(46.52) \\
D E=2.92\end{array}$ & $\begin{array}{c}18.00 \\
(47.89) \\
D E=2.67\end{array}$ & $\begin{array}{c}22.40 \\
(47.96) \\
D E=3.05\end{array}$ & $\begin{array}{c}24.00 \\
(46.68) \\
D E=1.51\end{array}$ & 0.5254 \\
\hline a* & $\begin{array}{c}23.75 \\
(9.61) \\
D E=1.44\end{array}$ & $\begin{array}{c}18.70 \\
(8.94) \\
D E=2.13\end{array}$ & $\begin{array}{c}22.05 \\
(9.26) \\
D E=1.84\end{array}$ & $\begin{array}{c}17.50 \\
(8.71) \\
+\mathrm{DE}=1.79\end{array}$ & 0.6053 \\
\hline b* & $\begin{array}{c}17.10 \\
(1.87) \\
D E=2.09\end{array}$ & $\begin{array}{c}24.05 \\
(3.22) \\
D E=1.60\end{array}$ & $\begin{array}{c}15.30 \\
(1.86) \\
D E=1.31\end{array}$ & $\begin{array}{c}25.55 \\
(3.16) \\
D E=1.18\end{array}$ & 0.1320 \\
\hline \multicolumn{6}{|c|}{$\begin{array}{l}\text { Pechuga } \\
\end{array}$} \\
\hline$L^{*}$ & $\begin{array}{c}16.70 \\
(47.63) \\
D E=2.49\end{array}$ & $\begin{array}{c}21.70 \\
(48.92) \\
D E=2.20\end{array}$ & $\begin{array}{c}23.10 \\
(49.18) \\
D E=3.10\end{array}$ & $\begin{array}{c}20.50 \\
(48.96) \\
D E=3.50\end{array}$ & 0.6466 \\
\hline a* & $\begin{array}{c}27.45 \\
(6.80) \\
D E=0.51\end{array}$ & $\begin{array}{c}14.90 \\
(5.71) \\
D E=1.41\end{array}$ & $\begin{array}{c}22.20 \\
(6.57) \\
D E=1.39\end{array}$ & $\begin{array}{c}17.45 \\
(5.89) \\
D E=1.08\end{array}$ & 0.0813 \\
\hline$b^{*}$ & $\begin{array}{c}17.70 \\
(6.31) \\
D E=1.61\end{array}$ & $\begin{array}{c}25.40 \\
(7.23) \\
D E=1.70\end{array}$ & $\begin{array}{c}18.20 \\
(6.31) \\
D E=2.02\end{array}$ & $\begin{array}{c}20.70 \\
(6.58) \\
D E=1.63\end{array}$ & 0.4368 \\
\hline
\end{tabular}

$\mathrm{P}=$ Palmiche; $\mathrm{a}, \mathrm{b}$ : letras distintas indican diferencias significativas para $\mathrm{p}<0.05$ (8) () Medias originales
En las tablas 7 a la 10 se muestran los resultados de composición de ácidos grasos en muslo, pierna, pechuga y grasa abdominal de pollos de ceba que consumen harina de palmiche, respectivamente. De manera general, se observó aumento de los ácidos láurico y mirístico, en tanto que palmítico, palmitoleico y oleico disminuyeron con respecto al control y linoleico y linolénico no difirieron entre tratamientos.

Tabla 7. Ácidos grasos en el muslo de pollos de ceba que consumen harina de palmiche.

\begin{tabular}{|c|c|c|c|c|c|c|}
\hline \multirow{2}{*}{$\begin{array}{c}\text { Ácido } \\
\text { graso (\%) }\end{array}$} & \multicolumn{4}{|c|}{ Tratamientos } & \multirow{2}{*}{ EE \pm} & \multirow{2}{*}{ p-valor } \\
\hline & Control & P 5\% & P $10 \%$ & P $15 \%$ & & \\
\hline $\begin{array}{l}\text { Láurico } \\
(12: 0)\end{array}$ & $0.30^{a}$ & $1.10^{\mathrm{b}}$ & $1.66^{\mathrm{bc}}$ & $2.06^{c}$ & 0.18 & 0.0005 \\
\hline $\begin{array}{l}\text { Mirístico } \\
(14: 0)\end{array}$ & $0.79^{a}$ & $1.52^{b}$ & $1.89^{b c}$ & $2.16^{c}$ & 0.14 & 0.0007 \\
\hline $\begin{array}{l}\text { Palmítico } \\
(16: 0)\end{array}$ & $21.54^{\mathrm{b}}$ & $21.29^{\mathrm{b}}$ & $20.67 \mathrm{ab}$ & $19.71^{a}$ & 0.42 & 0.0582 \\
\hline $\begin{array}{l}\text { Palmitoleico } \\
(16: 1)\end{array}$ & $5.90^{b}$ & $4.12^{a}$ & $4.26^{a}$ & $4.06^{a}$ & 0.32 & 0.0104 \\
\hline $\begin{array}{l}\text { Oleico } \\
(18: 1)\end{array}$ & $39.65^{b}$ & $37.79^{a b}$ & b $36.83^{a}$ & $35.67^{a}$ & 0.70 & 0.0215 \\
\hline $\begin{array}{l}\text { Linoleico } \\
(18: 2)\end{array}$ & 23.12 & 25.13 & 26.12 & 27.47 & 1.17 & 0.1388 \\
\hline $\begin{array}{c}\text { Linolénico } \\
(18: 3)\end{array}$ & 2.11 & 1.91 & 2.12 & 2.26 & 0.16 & 0.5062 \\
\hline
\end{tabular}

$\mathrm{P}=$ Palmiche; $\mathrm{a}, \mathrm{b}:$ Letras distintas indican diferencias significativas para $p<0.5062(7)$.

Tabla 8. Ácidos grasos en la pierna de pollos de ceba que consumen harina de palmiche.

\begin{tabular}{cccccccc}
\hline $\begin{array}{c}\text { Ácido } \\
\text { graso (\%) Control P 5\% P 10\% P 15\% }\end{array}$ & EE士 & p-valor \\
\hline $\begin{array}{c}\text { Láurico } \\
(12: 0)\end{array}$ & $0.22^{\mathrm{a}}$ & $1.01^{\mathrm{b}}$ & $1.86^{\mathrm{c}}$ & $2.18^{\mathrm{c}}$ & 0.14 & $<0.0001$ \\
$\begin{array}{c}\text { Mirístico } \\
(14: 0)\end{array}$ & $0.70^{\mathrm{a}}$ & $1.37^{\mathrm{b}}$ & $2.16^{\mathrm{c}}$ & $2.24^{\mathrm{c}}$ & 0.05 & $<0.0001$ \\
$\begin{array}{c}\text { Palmítico } \\
(16: 0)\end{array}$ & 22.43 & 22.36 & 21.56 & 21.33 & 0.47 & 0.3209 \\
$\begin{array}{c}\text { Palmitoleico } \\
(16: 1)\end{array}$ & $5.27^{\mathrm{b}}$ & $3.69^{\mathrm{a}}$ & $3.82^{\mathrm{a}}$ & $3.40^{\mathrm{a}}$ & 0.31 & 0.0105 \\
$\begin{array}{c}\text { Oleico } \\
(18: 1)\end{array}$ & 39.51 & 37.08 & 36.72 & 36.42 & 0.79 & 0.0843 \\
$\begin{array}{c}\text { Linoleico } \\
(18: 2)\end{array}$ & 23.30 & 25.09 & 25.21 & 25.52 & 1.30 & 0.6380 \\
$\begin{array}{c}\text { Linolénico } \\
(18: 3)\end{array}$ & 1.91 & 1.95 & 2.04 & 1.87 & 0.16 & 0.8968 \\
\hline
\end{tabular}

$\mathrm{P}=$ Palmiche; $\mathrm{a}, \mathrm{b}:$ letras distintas indican diferencias significativas para $p<0.05(7)$. 
Tabla 9. Ácidos grasos en la pechuga de pollos de ceba que consumen harina de palmiche.

\begin{tabular}{|c|c|c|c|c|c|c|}
\hline \multirow{2}{*}{$\begin{array}{c}\text { Ácido graso } \\
(\%)\end{array}$} & \multicolumn{4}{|c|}{ Tratamientos } & \multirow{2}{*}{ EE士 } & \multirow{2}{*}{ p-valor } \\
\hline & Control & P 5\% & P $10 \%$ & P 15\% & & \\
\hline $\begin{array}{l}\text { Láurico } \\
(12: 0)\end{array}$ & $0.31^{a}$ & $1.20^{\mathrm{b}}$ & $1.97^{c}$ & $2.59^{c}$ & 0.21 & 0.0003 \\
\hline $\begin{array}{l}\text { Mirístico } \\
(14: 0)\end{array}$ & $0.85^{a}$ & $1.59^{b}$ & $1.99^{b}$ & $2.66^{c}$ & 0.17 & 0.0005 \\
\hline $\begin{array}{l}\text { Palmítico } \\
(16: 0)\end{array}$ & $24.39^{c}$ & $23.77^{b c}$ & $21.83^{a}$ & $22.43^{a b}$ & 0.50 & 0.0239 \\
\hline $\begin{array}{c}\text { Palmitoleico } \\
(16: 1)\end{array}$ & $5.89^{b}$ & $4.19^{a}$ & $3.87^{a}$ & $3.96^{\mathrm{a}}$ & 0.31 & 0.0053 \\
\hline Oleico $(18: 1)$ & $43.10^{b}$ & $39.71^{a}$ & $38.77^{a}$ & $39.54^{a}$ & 0.68 & 0.0085 \\
\hline $\begin{array}{l}\text { Linoleico } \\
(18: 2)\end{array}$ & 17.29 & 20.66 & 23.29 & 20.41 & 1.44 & 0.1003 \\
\hline $\begin{array}{c}\text { Linolénico } \\
(18: 3)\end{array}$ & 1.33 & 1.55 & 1.66 & 1.61 & 0.19 & 0.6574 \\
\hline
\end{tabular}

$\mathrm{P}=$ Palmiche; $\mathrm{a}, \mathrm{b}:$ Letras distintas indican diferencias significativas para $\mathrm{p}<0.05(7)$.

Tabla 10. Ácidos grasos en la grasa abdominal de pollos de ceba que consumen harina de palmiche.

\begin{tabular}{|c|c|c|c|c|c|c|}
\hline \multirow{2}{*}{$\begin{array}{l}\text { Ácido graso } \\
(\%)\end{array}$} & \multicolumn{4}{|c|}{ Tratamientos } & \multirow{2}{*}{ EE士 } & \multirow{2}{*}{ P-valor } \\
\hline & Control & I P 5\% & P $10 \%$ & P 15\% & & \\
\hline $\begin{array}{l}\text { Láurico } \\
(12: 0)\end{array}$ & $0.43^{a}$ & $1.03^{a}$ & $2.40^{\mathrm{b}}$ & $2.68^{b}$ & 0.20 & 0.0001 \\
\hline $\begin{array}{c}\text { Mirístico } \\
(14: 0)\end{array}$ & $0.86^{a}$ & $1.41^{\mathrm{b}}$ & $2.50^{c}$ & $2.55^{c}$ & 0.14 & 0.0001 \\
\hline $\begin{array}{c}\text { Palmítico } \\
(16: 0)\end{array}$ & $22.93^{c}$ & $22.77^{\mathrm{bc}}$ & $21.85^{\mathrm{ab}}$ & $21.46^{a}$ & 0.30 & 0.0227 \\
\hline $\begin{array}{l}\text { Palmitoleico } \\
\quad(16: 1)\end{array}$ & $5.63^{b}$ & $4.83^{a b}$ & $4.15^{\mathrm{a}}$ & $4.24^{a}$ & 0.33 & 0.0477 \\
\hline Oleico (18:1) & $42.02^{b}$ & $39.31^{\mathrm{a}}$ & $39.51^{a}$ & $38.50^{a}$ & 0.52 & 0.0074 \\
\hline $\begin{array}{l}\text { Linoleico } \\
(18: 2)\end{array}$ & 20.44 & 23.11 & 21.83 & 22.88 & 0.90 & 0.2186 \\
\hline $\begin{array}{c}\text { Linolénico } \\
(18: 3)\end{array}$ & 1.58 & 1.45 & 1.56 & 1.46 & 0.10 & 0.7153 \\
\hline
\end{tabular}

$\mathrm{P}=$ Palmiche; $\mathrm{a}, \mathrm{b}$ : Letras distintas indican diferencias significativas para $p<0.05$ (7).

\section{DISCUSIÓN}

La no detección de diferencias entre tratamientos para los pesos vivo final y relativo de la canal indica que el empleo del palmiche en la dieta de pollos de ceba hasta el $15 \%$, no incide en los indicadores. Por tal razón, puede considerarse una fuente no convencional de alimentos localmente disponible que puede utilizarse para las aves.

La reducción de la grasa abdominal pudiera explicarse por los altos valores de fibra que se reportan en la harina de la oleaginosa ya que esta fracción en las raciones de las aves tiene un efecto reductor a nivel intestinal de la absorción del colesterol y de los lípidos $(10,11)$. Según Caro et al (12) y Arias et al (13), la harina de palmiche posee altos valores de FDN: $53.98 \%$, FDA: $45.48 \%$, lignina: $10.70 \%$ y FB: $45.92 \%$, respectivamente.

Según Petracci et al (14) antes del sacrifico la carne aviar posee $\mathrm{pH} 7$ y disminuye entre 5.8 y 6 a partir de las 6 horas postmortem, en el cual desarrolla rigidez (rigor mortis). La inclusión de harina de palmiche en la ración no modificó el indicador, por lo que este comportamiento quizás puede estar relacionado con las características genéticas del híbrido que se empleó en el estudio. Attia et al (15) plantearon que la tasa de disminución varía entre líneas genéticas y que los valores normales se encuentran entre 6,2 a 6,6 como los reportados en el presente experimento.

El color de la carne se correlaciona con el $\mathrm{pH}$ del músculo (16). Esto se debe a que en la carne, cuando el pH está por encima del punto isoeléctrico de las proteínas miofibrilares, las moléculas de agua se unen fuertemente por lo que se absorbe más luz en el músculo y se torna más oscuro (17). Un pH alto aumenta la importancia de la mioglobina en la absorción selectiva de luz verde, lo que da como resultado una carne que aparece más roja y menos amarilla (18), como se observó en el presente estudio.

Las aves que se alimentaron con harina de palmiche, depositaron los ácidos oleico, linoleico y palmítico en mayor concentración con relación al resto de los $A G$ que se estudiaron en las diferentes porciones. Resultados similares reportaron Lara et al (19) cuando estudiaron diferentes fuentes de lípidos en la dieta de pollos de ceba. Según Khalifa et al (20) el ácido oleico se asocia con la disminución del riesgo de enfermedades cardiovasculares al reducir los niveles séricos de colesterol por el descenso en LDL colesterol. El ácido linoleico además, se asocia en humanos con acción anticarcinogénica, mejora de la función inmune y reducción de la grasa corporal así como previene la ateroesclerosis (21). Estos resultados demuestran que al emplear el ingrediente en la alimentación de pollos de ceba hasta el $15 \%$, se obtienen canales magras y saludables para el consumo humano, todo lo cual le confiere valor agregado a las carnes. 
El aumento en la concentración de los ácidos saturados y la reducción de los insaturados con el aumento del palmiche en la ración puede explicarse por las proporciones de estos en la dieta y/o por su síntesis en el hígado. Varios autores plantean que la composición de ácidos grasos en las canales se modifica de acuerdo al perfil de ácidos grasos en la fuente que se adiciona a la dieta $(1,22)$. Según Rodríguez et al (3) en el aceite que se extrae del fruto completo de la palma real cubana los ácidos individuales en mayor proporción son el oleico (32.9\%), láurico $(23.3 \%)$, linoleico $(15.4 \%)$, palmítico (12.6\%) y mirístico $(11.0 \%)$.

Por otra parte, según Ajantha et al (21) y Toomer et al (23) se demostró que en el hígado se modifica la actividad del complejo hepático 9-desaturasa encargado de la síntesis de ácidos grasos saturados durante la digestión de los ácidos grasos insaturados, lo que repercute en la composición de la grasa corporal en aves. Además, el ácido linoleico puede decrecer las concentraciones de ácido oleico y palmitoleico en pollos de ceba (24).
Se concluye que la inclusión de harina de palmiche a las dietas de pollos de ceba no modifica el rendimiento de la canal, reduce la grasa abdominal e incide positivamente en los indicadores de calidad de la carne.

\section{Conflicto de intereses}

Los autores del presente estudio declaramos que no existe conflicto de intereses con la publicación de este manuscrito.

\section{Agradecimientos}

Los autores agradecen al Centro de Procesamiento cárnico del Instituto de Ciencia Animal, en especial al obrero Georvis Ojeda Santana y al técnico Fidel Monteagudo por la ayuda brindada en el procesamiento de las diferentes secciones de la canal.

\section{REFERENCIAS}

1. Akbar Mir N, Rafiq A, Kumar F, Singh V, Shukla V. Determinants of broiler chicken meat quality and factors affecting them: a review. J Food Sci Technol. 2017; 54(10):29973009. https://doi.org/10.1007/s13197$\underline{017-2789-z}$

2. Oliva $D$, Martínez $M$, Jiménez $L$, Ly $J$. Performance traits of growing pigs fed on diets of royal palm nut meal. Cuban J Agric Sci. 2018; 52(2):1-8. http://www. cjascience.com/index.php/CJAS/article/ view/793

3. Rodríguez E, Vicent R, González V, Adames Y, Tirado S, Lightbourne E. Obtención de aceite del fruto completo de Roystonea regia con diferentes disolventes. Rev Cub Química. 2011; 23(3):34-38. https://www.redalyc. org/pdf/4435/443543724004.pdf

4. Marrero D, Morales C L, Rodríguez E A, González V. Determination of sterol and fatty alcohols in unsaponifiable matter of Roystonea regia fruits oil. J Med Plant Res. 2013; 7(37):2736-2740. https://academicjournals. org/article/article1380721933 Delange\%20 et\%20al.pdf
5. Wideman N, O'Bryan CA, Crandall PG. Factors affecting poultry meat colour and consumer preferences. A review. World Poultry Sci J. 2016; 72(2):353-366. https:// doi.org/10.1017/S0043933916000015

6. NRC (National Research Council). Nutrient Requirements of Poultry. Ninth revised edition. Washington D.C: Editorial National Academic Press; 1994.

7. Duncan DB. Multiple Range and Multiple $F$ Tests. Biometrics. 1955; 11(1):1-42. https://doi.org/10.2307/3001478

8. Conover WJ. Some tests based on the binomial distribution. Practical nonparametric statistics, 3rd ed. John Wiley and Sons, Inc: New York, NY; 1999.

9. Di Rienzo JA, Casanoves F, Balzarini MG, Gonzalez L, Tablada M, Robledo CW. InfoStat. Grupo InfoStat, FCA, Universidad Nacional de Córdoba, Argentina. 2012. URL http://www.infostat.com.ar 
10. Santos M, Lon-Wo E, Savón L, Herrera M. Inclusion of Morus alba leaf meal: its effect on apparent retention of nutrient, productive performance and quality of the carcass of naked neck fowls. Cuban J Agric Sci. 2014; 48(3):265-269. http://www.cjascience. com/index.php/CJAS/article/view/581

11. Ashayerizadeh A, Dastar B, Shams Shargh M, Sadeghi Mahoonak A, Zerehdaran S. Effects of feeding fermented rapeseed meal on growth performance, gastrointestinal microflora population, blood metabolites, meat quality, and lipid metabolism in broiler chickens. Livestock Science. 2018; 216:183-190. https://doi.org/10.106/j. livsci.2018.08.012

12. Caro $Y$, Bustamante $D$, Arias $R$, Batista $R$, Pérez N, Contino $Y$, Almaguel R, Castro M, Ly J. Estudios de la composición química de palmiches cubanos destinados a alimentar ganado porcino y cunícula. Revista Computadorizada de Producción Porcina (RCPP). 2015; 22(2):79-81. http://www. iip.co.cu/RCPP/222/222 03YCaro.pdf

13. Arias $R$, Reyes $J \mathrm{~L}$, Bustamante $\mathrm{D}$, Jiménez L, Caro Y, Ly J. Caracterización química e índices químico-físicos de palmiches artemiseños para cerdos. Livest Res Rural Dev. 2016; 28(3). http://www.Irrd.org/ Irrd28/3/aria28036.html

14. Petracci M, Mudalal S, Soglia F, Cavani C. Meat quality in fast-growing broiler chickens. World Poultry Sci j. 2015. 71(2):363-374. https://doi.org/10.1017/ $\underline{\text { S0043933915000367 }}$

15. Attia YA, Al-Harthi MA, Korish MA, Shiboob MM. Evaluación de la calidad de la carne de pollo en el mercado minorista: efectos del tipo y origen de las canales. Rev Mex Cienc. Pecu. 2016; 7(3):321-339. https:// doi.org/10.22319/rmcp.v7i3.4213

16. Velasco $V$, Soto $V H$, Williams $P$, Campos J, Astudillo R, Rodríguez H. Meat quality parameters of broiler chickens fed diets containing chicory (Cichorium intybus) vinasse. Chilean J. Agric. Anim. Sci., ex AgroCiencia 2018; 34(1):26-32. http://dx.doi. org/10.4067/SO719-38902018005000203

17. Rajkumar U, Muthukumar M, Haunshi S, Niranjan M, Raju MVLN, Rama Rao SV, Chatterjee RN. Comparative evaluation of carcass traits and meat quality in native Aseel chickens and commercial broilers. Br Poult Sci. 2016; 57(3):339-347. http://dx.doi.or $\mathrm{g} / 10.1080 / 00071668.2016 .1162282$
18. Džinić N, Puvača N, Tasić $T$, Ikonić $P$, Okanović Đ. How meat quality and sensory perception is influenced by feeding poultry plant extracts. World Poultry Sci J. 2015; 71(4):673-681. https://doi.org/10.1017/ $\underline{\mathrm{S} 0043933915002378}$

19. Lara LJC, Baião NC, Aguilar CAL, Cançado SV, Fiuza MA, Ribeiro BRC. Rendimento, composição e teor de ácidos graxos da carcaça de frangos de corte alimentados com diferentes fontes lipídicas. Arq Bras Med Vet Zootec. 2006; 58(1):108-115. https://doi. org/10.1590/S0102-09352006000100016

20. Khalifa AH, Omar MB, Hussein SM, Abdelmbdy HE. Nutritional Value of Farmed and Wild Quail Meats. Assiut. J Agric Sci. 2016; 47(6-1):58-71. https://doi.org/10.21608/ ajas. 2016.2574

21. Ajantha A, Senthilkumar S, Sakthivael PC, Purushothaman MR. Nutritional influence on quality of egg and meat in poultry. A Review Int J Environ Sci Technol. 2017; 6(6):33383345. http://ijset.net/journal/1968.pdf

22. Avazkhanloo M, Shahir MH, Khalaji S, JafariAnarkooli I. Flaxseed extrusion and expansion coupled with enzyme and pelleting changed protein and lipid molecular structure of flaxseed and improved digestive enzymes activity, intestinal morphology, breast muscle fatty acids and performance of broiler chickens. Anim. Feed Sci. Technol. 2020; 260: Article114341. https://doi. org/10.1016/j.anifeedsci.2019.114341

23. Toomer OT, Livingston $M$, Wall $B$, Sanders $\mathrm{E}, \mathrm{Vu}$ T, Malheiros, RD, et al. Feeding higholeic peanuts to meat-type broiler chickens enhances the fatty acid profile of the meat produced. Poult Sci J. 2020; 99(4): 2236-2245. https://doi.org/10.1016/j. psj.2019.11.015

24. Kumari S, Meng GY, Ebrahimi M. Conjugated linoleic acid as functional food in poultry products: A review. Int J Food Prop. 2017; 20(3):491-506. https://doi.org/10.1080/1 $\underline{0942912.2016 .1168835}$ 